\title{
Multiple Positive Solutions for Singular Quasilinear Multipoint BVPs with the First-Order Derivative
}

\author{
Weihua Jiang, ${ }^{1,2}$ Bin Wang, ${ }^{3}$ and Yanping Guo ${ }^{1}$ \\ ${ }^{1}$ College of Sciences, Hebei University of Science and Technology, Shijiazhuang, 050018 Hebei, China \\ ${ }^{2}$ College of Mathematics and Science of Information, Hebei Normal University, Shijiazhuang, \\ 050016 Hebei, China \\ ${ }^{3}$ Department of Basic Courses, Hebei Professional and Technological College of Chemical and \\ Pharmaceutical Engineering, Shijiazhuang, 050031 Hebei, China
}

Correspondence should be addressed to Weihua Jiang, weihuajiang@hebust.edu.cn

Received 28 November 2007; Accepted 1 April 2008

Recommended by Wenming Zou

The existence of at least three positive solutions for differential equation $\left(\phi_{p}\left(u^{\prime}(t)\right)\right)^{\prime}+g(t) f(t$, $\left.u(t), u^{\prime}(t)\right)=0$, under one of the following boundary conditions: $u(0)=\sum_{i=1}^{m-2} a_{i} u\left(\xi_{i}\right), \varphi_{p}\left(u^{\prime}(1)\right)=$ $\sum_{i=1}^{m-2} b_{i} \varphi_{p}\left(u^{\prime}\left(\xi_{i}\right)\right)$ or $\varphi_{p}\left(u^{\prime}(0)\right)=\sum_{i=1}^{m-2} a_{i} \varphi_{p}\left(u^{\prime}\left(\xi_{i}\right)\right), u(1)=\sum_{i=1}^{m-2} b_{i} u\left(\xi_{i}\right)$ is obtained by using the H. Amann fixed point theorem, where $\varphi_{p}(s)=|s|^{p-2} s, p>1,0<\xi_{1}<\xi_{2}<\cdots<\xi_{m-2}<1, a_{i}>0, b_{i}>0$, $0<\sum_{i=1}^{m-2} a_{i}<1,0<\sum_{i=1}^{m-2} b_{i}<1$. The interesting thing is that $g(t)$ may be singular at any point of $[0,1]$ and $f$ may be noncontinuous.

Copyright (c) 2008 Weihua Jiang et al. This is an open access article distributed under the Creative Commons Attribution License, which permits unrestricted use, distribution, and reproduction in any medium, provided the original work is properly cited.

\section{Introduction}

In this paper, of multiple positive solutions for differential equation

$$
\left(\varphi_{p}\left(u^{\prime}(t)\right)\right)^{\prime}+g(t) f\left(t, u(t), u^{\prime}(t)\right)=0, \quad \text { a. e. } t \in(0,1),
$$

subject to boundary conditions:

$$
\begin{aligned}
& u(0)=\sum_{i=1}^{m-2} a_{i} u\left(\xi_{i}\right), \quad \varphi_{p}\left(u^{\prime}(1)\right)=\sum_{i=1}^{m-2} b_{i} \varphi\left(u^{\prime}\left(\xi_{i}\right)\right), \\
& \varphi_{p}\left(u^{\prime}(0)\right)=\sum_{i=1}^{m-2} a_{i} \varphi_{p}\left(u^{\prime}\left(\xi_{i}\right)\right), \quad u(1)=\sum_{i=1}^{m-2} b_{i} u\left(\xi_{i}\right),
\end{aligned}
$$


respectively, where $\varphi_{p}(s)=|s|^{p-2} s, p>1,0<\xi_{1}<\xi_{2}<\cdots<\xi_{m-2}<1, a_{i}>0, b_{i}>0,0<$ $\sum_{i=1}^{m-2} a_{i}<1,0<\sum_{i=1}^{m-2} b_{i}<1, g(t)$ may be singular at any point of $[0,1]$.

The multipoint boundary value problems for ordinary differential equations arise in a variety of different areas of applied mathematics and physics. The study of the multipoint boundary value problems for linear second-order ordinary differential equations was initiated by Il'in and Moiseev [1, 2]. Since then, nonlinear second-order multipoint boundary value problems have been studied by several authors. We refer the reader to [3-9] and references cited therein. Recently, in [10], Liang and Zhang studied the existence of positive solutions for differential equation

$$
\left(\varphi\left(u^{\prime}\right)\right)^{\prime}+a(t) f(u(t))=0, \quad 0<t<1,
$$

under the boundary conditions (1.2) by using the fixed point index theory. Wang and Hou [11] investigated the multiplicity of solutions for the differential equation

$$
\left(\varphi_{p}\left(u^{\prime}(t)\right)\right)^{\prime}+f(t, u(t))=0, \quad t \in(0,1),
$$

under the boundary conditions (1.3) by utilizing the fixed point theorem for operators on a cone. Guo et al. [12] proved the existence of at least three positive solutions for differential equation

$$
\left(\varphi_{p}\left(u^{\prime}(t)\right)\right)^{\prime}+a(t) f(t, u(t))=0, \quad t \in(0,1),
$$

subject to (1.2) and (1.3), respectively, by using the five-functional-fixed-point theorem, where $a(t)$ and $f(t, u)$ are continuous.

All of the above work was done under the assumption that $f$ is allowed to depend just on $u$, while the first-order derivative $u^{\prime}(t)$ is not involved explicitly in the nonlinear term $f$.

In $[13,14]$, Wang and Ge and Sun et al. proved the existence of multiple positive solutions for (1.1) subject to boundary conditions:

$$
\begin{aligned}
u(0) & =\sum_{i=1}^{n-2} \alpha_{i} u\left(\xi_{i}\right), & u^{\prime}(1) & =\sum_{i=1}^{n-2} \beta_{i} u^{\prime}\left(\xi_{i}\right), \\
u^{\prime}(0) & =\sum_{i=1}^{n} \alpha_{i} u^{\prime}\left(\xi_{i}\right), & u(1) & =\sum_{i=1}^{n} \beta_{i} u\left(\xi_{i}\right),
\end{aligned}
$$

respectively, where $g$ and $f$ are continuous.

However, in the existing literature, few people considered the case where the nonlinear term is not only involved in the first-order derivative but also noncontinuous. Our paper will fill this gap in the literature. The purpose of this paper is to improve and generalize the results in the above mentioned references. We will prove that the problem (1.1), (1.2) and the problem (1.1), (1.3) have at least three positive solutions by using the H. Amann fixed point theorem, where $g(t)$ may be singular at any point of $[0,1]$ and $f(t, u, v)$ may be noncontinuous.

In this paper, we always suppose the following conditions are satisfied:

$$
\begin{aligned}
& \left(\mathrm{H}_{1}\right) 0=\xi_{0}<\xi_{1}<\xi_{2}<\cdots<\xi_{m-2}<\xi_{m-1}=1, a_{i}>0, b_{i}>0,0<\sum_{i=1}^{m-2} a_{i}<1,0<\sum_{i=1}^{m-2} b_{i}<1 ; \\
& \left(\mathrm{H}_{2}\right) g(t) \in L^{1}[0,1], g(t)>0, \text { a.e. } t \in[0,1] ;
\end{aligned}
$$


Weihua Jiang et al.

$\left(\mathrm{H}_{3}\right) f(t, u, v):[0,1] \times R \times R \rightarrow[0, \infty)$ is bounded in a bounded subset of $[0,1] \times R \times R$, $f(t, u(t), v(t))$ is measurable for $u(t), v(t) \in C[0,1]$, and $f(t, \cdot, \cdot)$ is continuous for a.e. $t \in[0,1]$.

Sometimes, we will make use of the following conditions.

$\left(\mathrm{H}_{4}\right) f(t, \cdot, v)$ and $f(t, u, \cdot)$ are strictly increasing in $[0, \infty)$.

$\left(\mathrm{H}_{5}\right) f(t, \cdot, v)$ is strictly increasing in $[0, \infty)$ and $f(t, u, \cdot)$ is strictly decreasing in $(-\infty, 0]$.

\section{Preliminaries}

Definition 2.1. Let $X$ be a real Banach space and $P \subset X$ be a cone. For $u, v \in X$, we denote

$$
\begin{gathered}
u \preccurlyeq v \Longleftrightarrow u-v \in P, \\
u \prec v \Longleftrightarrow u-v \in P, \quad \text { but } u \neq v, \\
u \prec v \Longleftrightarrow u-v \in \operatorname{int} P .
\end{gathered}
$$

If $P$ has nonempty interior, then it is called a solid cone. If every ordered interval is bounded, then $P$ is called a normal cone.

Definition 2.2. An operator $T: P \rightarrow X$ is called order preserving if $u \preccurlyeq v \Rightarrow T u \preccurlyeq T v$, strictly order preserving if $u \prec v \Rightarrow T u \prec T v$, and strongly order preserving if $u \prec \prec v \Rightarrow T u \prec \prec T v$.

Lemma 2.3 (see $[15,16]$ ). Let $P$ be a normal solid cone of a real Banach space $X$ and suppose there exist $y_{1}, z_{1}, y_{2}, z_{2} \in X$ such that $y_{1} \prec z_{1} \prec y_{2} \prec z_{2}$. In addition, suppose that $T:\left[y_{1}, z_{2}\right] \rightarrow X$ is a completely continuous and strongly order preserving operator such that

$$
y_{1} \preccurlyeq T y_{1}, \quad T z_{1} \prec z_{1}, \quad y_{2} \prec T y_{2}, \quad T z_{2} \preccurlyeq z_{2} .
$$

Then $T$ has at least three fixed points $x_{1}, x_{2}$ and $x_{3}$ satisfying

$$
y_{1} \preccurlyeq x_{1} \prec \prec z_{1}, \quad y_{2} \prec \prec x_{2} \preccurlyeq z_{2}, \quad y_{2} \npreceq x_{3} \npreceq z_{1} .
$$

\section{The positive solutions for the problem (1.1), (1.2)}

Let $X=C^{1}[0,1]$ with norm $\|u\|=\max \left\{\max _{t \in[0,1]}|u(t)|, \max _{t \in[0,1]}\left|u^{\prime}(t)\right|\right\}$. Define $P \subset X$ by

$$
P=\left\{u \in X \mid u(t) \geq 0, u^{\prime}(t) \geq 0, t \in[0,1]\right\} .
$$

Obviously, $X$ is a Banach space and $P$ is a normal solid cone of $X$.

We can easily get the following lemmas.

Lemma 3.1. The boundary value problem (1.1), (1.2) has a solution $u(t)$ if and only if $u(t)$ satisfies the equation

$$
u(t)=\int_{0}^{t} \varphi_{q}\left(\int_{s}^{1} g(\tau) f\left(\tau, u(\tau), u^{\prime}(\tau)\right) d \tau+B f\right) d s+A f
$$


where $\varphi_{q}$ is the inverse function of $\varphi_{p}$, that is, $\varphi_{q}=\varphi_{p}^{-1}, 1 / p+1 / q=1$, and

$$
\begin{aligned}
& B u=\frac{1}{1-\sum_{i=1}^{m-2} b_{i}} \sum_{i=1}^{m-2} b_{i} \int_{\xi_{i}}^{1} g(\tau) u(\tau) d \tau, \quad u \in L^{\infty}[0,1] \\
& A u=\frac{1}{1-\sum_{i=1}^{m-2} a_{i}} \sum_{i=1}^{m-2} a_{i} \int_{0}^{\xi_{i}} \varphi_{q}\left(\int_{s}^{1} g(\tau) u(\tau) d \tau+B u\right) d s, \quad u \in L^{\infty}[0,1] .
\end{aligned}
$$

Define an operator $T$ in $P$ by

$$
T u(t)=\int_{0}^{t} \varphi_{q}\left(\int_{s}^{1} g(\tau) f\left(\tau, u(\tau), u^{\prime}(\tau)\right) d \tau+B f\right) d s+A f
$$

Evidently, $u(t)$ is a fixed point of $T$ if and only if it is a solution of the problem (1.1), (1.2).

Lemma 3.2. Suppose $\left(H_{1}\right)-\left(H_{4}\right)$ hold, $u(t) \in P$, then $T u(t) \geq 0,(T u)^{\prime}(t) \geq 0, t \in[0,1]$.

For convenience, we denote

$$
L u(t)=\int_{0}^{t} \varphi_{q}\left(\int_{s}^{1} g(\tau) u(\tau) d \tau+B u\right) d s+A u, \quad u \in L^{\infty}[0,1]
$$

Obviously, we have $T u(t)=L f\left(t, u(t), u^{\prime}(t)\right)$.

Lemma 3.3. Suppose $\left(H_{2}\right)$ holds. If $u_{1}(t), u_{2}(t) \in L^{\infty}[0,1]$ and $u_{1}(t) \leq(<) u_{2}(t)$, a.e.t $\in[0,1]$, then $L_{1} u_{1}(t) \leq(<) L_{1} u_{2}(t), t \in[0,1]$.

Assume $\left(\mathrm{H}_{1}\right)-\left(\mathrm{H}_{4}\right)$ hold. By Lemmas 3.2 and 3.3, the absolute continuity of integral, Ascoli-Arzela theorem and Lebesgue-Dominated-Convergence-theorem, we obtain that $T$ : $P \rightarrow P$ is completely continuous and $T$ is strongly order preserving.

Theorem 3.4. Suppose $\left(H_{1}\right)-\left(H_{4}\right)$ hold. In addition, suppose there exist constants $0<a<c<b<d$ and $u_{0}(t) \in P \backslash\{0\}$ satisfying $c L u_{0}(1)<b A u_{0}$ such that

$\left(\mathrm{A}_{1}\right) f\left(t, a A u_{0}, 0\right) \geq \varphi_{p}(a) u_{0}(t)$, a.e. $t \in[0,1]$;

$\left(\mathrm{A}_{2}\right) f\left(t, c L u_{0}(1), c\left(\operatorname{Lu}_{0}\right)^{\prime}(0)\right)<\varphi_{p}(c) u_{0}(t)$, a.e. $t \in[0,1]$;

$\left(\mathrm{A}_{3}\right) f\left(t, b A u_{0}, 0\right)>\varphi_{p}(b) u_{0}(t)$, a.e. $t \in[0,1]$

$\left(\mathrm{A}_{4}\right) f\left(t, d L u_{0}(1), d\left(L u_{0}\right)^{\prime}(0)\right) \leq \varphi_{p}(d) u_{0}(t)$, a.e. $t \in[0,1]$.

Then the problem (1.1), (1.2) has at least three positive solutions $u_{1}(t), u_{2}(t)$, and $u_{3}(t)$ satisfying

$$
\begin{aligned}
a L u_{0}(t) \preccurlyeq u_{1}(t) \prec & <c L u_{0}(t), \quad b L u_{0}(t) \prec<u_{2}(t) \preccurlyeq d L u_{0}(t), \\
& b L u_{0}(t) \npreceq u_{3}(t) \npreceq c L u_{0}(t) .
\end{aligned}
$$


Weihua Jiang et al.

Proof. Let $y_{1}(t)=a L u_{0}(t), z_{1}(t)=c L u_{0}(t), y_{2}(t)=b L u_{0}(t), z_{2}(t)=d L u_{0}(t)$. Obviously, we have

$$
y_{1}(t) \prec z_{1}(t) \prec y_{2}(t) \prec z_{2}(t)
$$

Firstly, we will show $y_{1}(t) \preccurlyeq T y_{1}(t)$.

By $y_{1}(t) \geq a A u_{0}, y_{1}^{\prime}(t) \geq 0,\left(\mathrm{~A}_{1}\right)$ and Lemma 3.3, we have

$$
\begin{aligned}
T y_{1}(t) & =L f\left(t, y_{1}(t), y_{1}^{\prime}(t)\right) \geq L f\left(t, a A u_{0}, 0\right) \geq a L u_{0}(t)=y_{1}(t) \\
\left(T y_{1}\right)^{\prime}(t) & =\varphi_{q}\left(\int_{t}^{1} g(\tau) f\left(\tau, y_{1}(\tau), y_{1}^{\prime}(\tau)\right) d \tau+B f\right) \geq a\left[\varphi_{q} \int_{t}^{1} g(\tau) u_{0}(\tau) d \tau+B u_{0}\right]=y_{1}^{\prime}(t) .
\end{aligned}
$$

So, we get $y_{1} \preccurlyeq T y_{1}$.

Similarly, by $\left(\mathrm{A}_{3}\right)$ and Lemma 3.3, we get $y_{2} \prec T y_{2}$.

Next, we prove $T z_{1} \prec z_{1}$.

From $z_{1}(t) \leq c L u_{0}(1), z_{1}^{\prime}(t) \leq c\left(L u_{0}\right)^{\prime}(0),\left(\mathrm{A}_{2}\right)$, and Lemma 3.3, we have

$$
\begin{aligned}
T z_{1}(t) & =L f\left(t, z_{1}(t), z_{1}^{\prime}(t)\right) \leq L f\left(t, c L u_{0}(1), c\left(L u_{0}\right)^{\prime}(0)\right)<c L u_{0}(t)=z_{1}(t) \\
\left(T z_{1}\right)^{\prime}(t) & =\varphi_{q}\left(\int_{t}^{1} g(\tau) f\left(\tau, z_{1}(\tau), z_{1}^{\prime}(\tau)\right) d \tau+B f\right) \leq c\left[\varphi_{q} \int_{t}^{1} g(\tau) u_{0}(\tau) d \tau+B u_{0}\right]=z_{1}^{\prime}(t) .
\end{aligned}
$$

So, we get $T z_{1} \prec z_{1}$.

Similarly, by $\left(\mathrm{A}_{4}\right)$ and Lemma 3.3, we get $T z_{2} \preccurlyeq z_{2}$.

By Lemma 2.3, we get that the operator $T$ has at least three fixed points $u_{1}(t), u_{2}(t)$, and $u_{3}(t)$ satisfying

$$
\begin{aligned}
a L u_{0}(t) \preccurlyeq u_{1}(t) \prec & <L u_{0}(t), \quad b L u_{0}(t) \prec \prec u_{2}(t) \preccurlyeq d L u_{0}(t), \\
& b L u_{0}(t) \npreceq u_{3}(t) \npreceq c L u_{0}(t) .
\end{aligned}
$$

The proof is completed.

\section{The positive solutions for the problem (1.1), (1.3)}

Let $X$ be the same as the one in Section 3. Define $P_{1} \subset X$ by

$$
P_{1}=\left\{u \in X \mid u(t) \geq 0, u^{\prime}(t) \leq 0, t \in[0,1]\right\} .
$$

Evidently, $P_{1}$ is a normal solid cone of $X$.

We can easily get the following lemmas.

Lemma 4.1. The boundary value problem (1.1), (1.3) has a solution $u(t)$ if and only if $u(t)$ satisfies the equation

$$
u(t)=-\int_{0}^{t} \varphi_{q}\left(\int_{0}^{s} g(\tau) f\left(\tau, u(\tau), u^{\prime}(\tau)\right) d \tau+\widetilde{B} f\right) d s+\tilde{A} f
$$


where $\varphi_{q}$ is the same as the one in Lemma 3.1, and

$$
\begin{aligned}
\widetilde{B} u=\frac{1}{1-\sum_{i=1}^{m-2} a_{i}} \sum_{i=1}^{m-2} a_{i} \int_{0}^{\xi_{i}} g(\tau) u(\tau) d \tau, \quad u \in L^{\infty}[0,1] \\
\tilde{A} u=\frac{1}{1-\sum_{i=1}^{m-2} b_{i}}\left[\int_{0}^{1} \varphi_{q}\left(\int_{0}^{s} g(\tau) u(\tau) d \tau+\widetilde{B} u\right) d s\right. \\
\left.\quad-\sum_{i=1}^{m-2} b_{i} \int_{0}^{\xi_{i}} \varphi_{q}\left(\int_{0}^{s} g(\tau) u(\tau) d \tau+\widetilde{B} u\right) d s\right], \quad u \in L^{\infty}[0,1] .
\end{aligned}
$$

Define an operator $T_{1}$ in $P_{1}$ by

$$
T_{1} u(t)=-\int_{0}^{t} \varphi_{q}\left(\int_{0}^{s} g(\tau) f\left(\tau, u(\tau), u^{\prime}(\tau)\right) d \tau+\tilde{B} f\right) d s+\tilde{A} f .
$$

Obviously, $u(t) \in P_{1}$ is a fixed point of the operator $T_{1}$ if and only if it is a positive solution of the problem (1.1), (1.3).

Lemma 4.2. Suppose $\left(H_{1}\right)-\left(H_{3}\right),\left(H_{5}\right)$ hold, and $u(t) \in P_{1}$, then $T_{1} u(t) \geq 0,\left(T_{1} u\right)^{\prime}(t) \leq 0, t \in[0,1]$.

For convenience, we denote

$$
L_{1} u(t)=-\int_{0}^{t} \varphi_{q}\left(\int_{0}^{s} g(\tau) u(\tau) d \tau+\widetilde{B} u\right) d s+\tilde{A} u .
$$

Clearly, $T_{1} u(t)=L_{1} f\left(t, u(t), u^{\prime}(t)\right)$.

Lemma 4.3. Suppose $\left(H_{2}\right)$ holds. If $u_{1}(t), u_{2}(t) \in L^{\infty}[0,1]$, and $u_{1}(t) \leq(<) u_{2}(t)$, a.e. $t \in[0,1]$, then $L_{1} u_{1}(t) \leq(<) L_{1} u_{2}(t), t \in[0,1]$.

Assume $\left(\mathrm{H}_{1}\right)-\left(\mathrm{H}_{3}\right)$ and $\left(\mathrm{H}_{5}\right)$ hold, by Lemmas 4.2 and 4.3 , the absolute continuity of integral, Ascoli-Arzela theorem, and Lebesgue-Dominated-Convergence-theorem, we obtain that $T_{1}: P_{1} \rightarrow P_{1}$ is completely continuous, and $T_{1}$ is strongly order preserving.

Theorem 4.4. Suppose $\left(H_{1}\right)-\left(H_{3}\right)$ and $\left(H_{5}\right)$ hold. In addition, suppose there exist constants $0<\tilde{a}<$ $\tilde{c}<\tilde{b}<\tilde{d}$ and function $u_{0}(t) \in P_{1} \backslash\{0\}$ satisfying $\tilde{c} \tilde{A} u_{0}<\tilde{b} L_{1} u_{0}(1)$ such that

$\left(C_{1}\right) f\left(t, \tilde{a} L_{1} u_{0}(1), 0\right) \geq \varphi_{p}(\tilde{a}) u_{0}(t)$, a.e. $t \in[0,1]$;

$\left(C_{2}\right) f\left(t, \tilde{c} \tilde{A} u_{0}, \widetilde{c}\left(L_{1} u_{0}\right)^{\prime}(1)\right)<\varphi_{p}(\widetilde{c}) u_{0}(t)$, a.e. $t \in[0,1]$;

$\left(C_{3}\right) f\left(t, \tilde{b} L_{1} u_{0}(1), 0\right)>\varphi_{p}(\tilde{b}) u_{0}(t)$, a.e. $t \in[0,1]$;

$\left(C_{4}\right) f\left(t, \tilde{d} \tilde{A} u_{0}, \tilde{d}\left(L_{1} u_{0}\right)^{\prime}(1)\right) \leq \varphi_{p}(\tilde{d}) u_{0}(t)$, a.e. $t \in[0,1]$.

Then the problem (1.1), (1.3) has at least three positive solutions $v_{1}(t), v_{2}(t)$, and $v_{3}(t)$ satisfying

$$
\begin{gathered}
\tilde{a} L_{1} u_{0}(t) \preccurlyeq v_{1}(t) \prec \prec \tilde{c} L_{1} u_{0}(t), \quad \tilde{b} L_{1} u_{0}(t) \prec \prec v_{2}(t) \preccurlyeq \tilde{d} L_{1} u_{0}(t), \\
\tilde{b} L_{1} u_{0}(t) \npreceq v_{3}(t) \npreceq \tilde{c} L_{1} u_{0}(t) .
\end{gathered}
$$


Weihua Jiang et al.

Proof. Let $y_{1}(t)=\tilde{a} L_{1} u_{0}(t), z_{1}(t)=\tilde{c} L_{1} u_{0}(t), y_{2}(t)=\tilde{b} L_{1} u_{0}(t), z_{2}(t)=\tilde{d} L_{1} u_{0}(t)$. Obviously, we have

$$
y_{1}(t) \prec z_{1}(t) \prec y_{2}(t) \prec z_{2}(t)
$$

Firstly, we will show $y_{1}(t) \preccurlyeq T_{1} y_{1}(t)$.

By $y_{1}(t) \geq \tilde{a} L_{1} u_{0}(1), y_{1}^{\prime}(t) \leq 0,\left(C_{1}\right)$, and Lemma 4.3 , we have

$$
\begin{aligned}
T_{1} y_{1}(t) & =L_{1} f\left(t, y_{1}(t), y_{1}^{\prime}(t)\right) \geq L_{1} f\left(t, \tilde{a} L_{1} u_{0}(1), 0\right) \geq \tilde{a} L_{1} u_{0}(t)=y_{1}(t), \\
\left(T_{1} y_{1}\right)^{\prime}(t) & =-\varphi_{q}\left(\int_{0}^{t} g(\tau) f\left(\tau, y_{1}(\tau), y_{1}^{\prime}(\tau)\right) d \tau+\widetilde{B} f\right) \\
& \leq-\tilde{a} \varphi_{q}\left[\int_{0}^{t} g(\tau) u_{0}(\tau) d \tau+\widetilde{B} u_{0}\right]=y_{1}^{\prime}(t) .
\end{aligned}
$$

So, we get $y_{1} \preccurlyeq T_{1} y_{1}$.

Similarly, by $\left(C_{3}\right)$ and Lemma 4.3 , we get $y_{2} \prec T_{1} y_{2}$.

Next, we will prove $T_{1} z_{1} \prec z_{1}$.

From $z_{1}(t) \leq \tilde{c} \tilde{A} u_{0}, z_{1}^{\prime}(t) \geq \widetilde{c}\left(L_{1} u_{0}\right)^{\prime}(1),\left(C_{2}\right)$, and Lemma 4.3 , we have

$$
\begin{aligned}
T_{1} z_{1}(t) & =L_{1} f\left(t, z_{1}(t), z_{1}^{\prime}(t)\right) \leq L_{1} f\left(t, \tilde{c} \tilde{A} u_{0}, \widetilde{c}\left(L_{1} u_{0}\right)^{\prime}(1)\right)<\widetilde{c} L_{1} u_{0}(t)=z_{1}(t), \\
\left(T_{1} z_{1}\right)^{\prime}(t) & =-\varphi_{q}\left(\int_{0}^{t} g(\tau) f\left(\tau, z_{1}(\tau), z_{1}^{\prime}(\tau)\right) d \tau+\widetilde{B} f\right) \\
& \geq-\tilde{c} \varphi_{q}\left[\int_{0}^{t} g(\tau) u_{0}(\tau) d \tau+\widetilde{B} u_{0}\right]=z_{1}^{\prime}(t) .
\end{aligned}
$$

So, we get $T_{1} z_{1} \prec z_{1}$.

Similarly, by $\left(C_{4}\right)$ and Lemma 4.3 , we get $T_{1} z_{2} \preccurlyeq z_{2}$.

By Lemma 2.3, we get that the operator $T_{1}$ has at least three fixed points $v_{1}(t), v_{2}(t)$, and $v_{3}(t)$ satisfying

$$
\begin{gathered}
\tilde{a} L_{1} u_{0}(t) \preccurlyeq v_{1}(t) \prec \prec \tilde{c} L_{1} u_{0}(t), \quad \tilde{b} L_{1} u_{0}(t) \prec \prec v_{2}(t) \preccurlyeq \tilde{d} L_{1} u_{0}(t), \\
\tilde{b} L_{1} u_{0}(t) \npreceq v_{3}(t) \npreceq \tilde{c} L_{1} u_{0}(t) .
\end{gathered}
$$

The proof is completed.

\section{Acknowledgments}

This work is supported by the Natural Science Foundation of China (10701032), Science and Technology Key Project of Hebei Province (07217169), and the Foundation of Hebei University of Science and Technology (XI200747). 


\section{References}

[1] V. A. Il'in and E. I. Moiseev, "Nonlocal boundary-value problem of the secod kind for a SturmLiouville operator," Differential Equations, vol. 23, no. 8, pp. 979-987, 1987.

[2] V. A. Il'in and E. I. Moiseev, "Nonlocal boundary value problem of the first kind for a Sturm-Liouville operator in its differential and finite-difference aspects," Differential Equations, vol. 23, no. 7, pp. 803810, 1987.

[3] H. Lü, D. O'Regan, and R. P. Agarwal, "Existence theorems for the one-dimensional singular pLaplacian equation with a nonlinear boundary condition," Journal of Computational and Applied Mathematics, vol. 182, no. 1, pp. 188-210, 2005.

[4] R. Ma, "Positive solutions of a nonlinear three-point boundary-value problem," Electronic Journal of Differential Equations, vol. 1999, no. 34, pp. 1-8, 1999.

[5] W. Jiang and F. Li, "Several existence theorems of monotone positive solutions for third-order multipoint boundary value problems," Boundary Value Problems, vol. 2007, Article ID 17951, 9 pages, 2007.

[6] Y. Guo and W. Ge, "Positive solutions for three-point boundary value problems with dependence on the first order derivative," Journal of Mathematical Analysis and Applications, vol. 290, no. 1, pp. 291-301, 2004.

[7] W. Jiang and Y. Guo, "Multiple positive solutions for second-order $m$-point boundary value problems," Journal of Mathematical Analysis and Applications, vol. 327, no. 1, pp. 415-424, 2007.

[8] W. Jiang, "Multiple positive solutions for $n$ th-order $m$-point boundary value problems with all derivatives," Nonlinear Analysis: Theory, Methods \& Applications, vol. 68, no. 5, pp. 1064-1072, 2008.

[9] W. Jiang, "Eigenvalue criteria for existence of multiple positive solutions of high-order nonlinear BVPs," Nonlinear Analysis: Theory, Methods \& Applications. In press.

[10] S. Liang and J. Zhang, "The existence of countably many positive solutions for nonlinear singular mpoint boundary value problems," Journal of Computational and Applied Mathematics, vol. 214, no. 1, pp. 78-89, 2008.

[11] Y. Wang and C. Hou, "Existence of multiple positive solutions for one-dimensional $p$-Laplacian," Journal of Mathematical Analysis and Applications, vol. 315, no. 1, pp. 144-153, 2006.

[12] Y. Guo, Y. Ji, and X. Liu, "Multiple positive solutions for multi-point boundary value problems with p-Laplacian," Journal of Computational and Applied Mathematics, vol. 216, no. 1, pp. 144-156, 2008.

[13] Y. Wang and W. Ge, "Multiple positive solutions for multipoint boundary value problems with onedimensional p-Laplacian," Journal of Mathematical Analysis and Applications, vol. 327, no. 2, pp. 1381$1395,2007$.

[14] B. Sun, W. Ge, and D. Zhao, "Three positive solutions for multipoint one-dimensional $p$-Laplacian boundary value problems with dependence on the first order derivative," Mathematical and Computer Modelling, vol. 45, no. 9-10, pp. 1170-1178, 2007.

[15] H. Amann, "Fixed point equations and nonlinear eigenvalue problems in ordered Banach spaces," SIAM Review, vol. 18, no. 4, pp. 620-709, 1976.

[16] D. Guo, Nonlinear Functional Analysis, Shandong Science and Technology Press, Jinan, China, 2004. 